\title{
DETECTION AND EXTRACTION OF WATER BOTTOM TOPOGRAPHY FROM LASERBATHYMETRY DATA BY USING FULL-WAVEFORM-STACKING TECHNIQUES
}

\author{
D.Mader $^{1, *}$ K. Richter ${ }^{1}$, P. Westfeld ${ }^{2}$, R. Wei $\beta^{3}$, H.-G. Maas ${ }^{1}$ \\ ${ }^{1}$ Institute of Photogrammetry and Remote Sensing, Technische Universität Dresden, Germany \\ (david.mader, katja.richter1, hans-gerd.maas)@tu-dresden.de \\ ${ }^{2}$ Federal Maritime and Hydrographic Agency (BSH), Section Geodetic-hydrographic Techniques and Systems, Germany \\ patrick.westfeld@bsh.de \\ ${ }^{3}$ German Federal Institute of Hydrology, Germany - weiss@bafg.de
}

Commission II - WG II/9

KEY WORDS: LiDAR, bathymetry, full-waveform processing, full-waveform-stacking

\begin{abstract}
:
Airborne LiDAR bathymetry allows an efficient and area-wide acquisition of water bottom points in shallow water areas. However, the measurement method is severely limited by water turbidity, impending a reliable detection of water bottom points at higher turbidity or in deeper water bodies. This leads to an incomplete acquisition of the water bottom topography. In this contribution, advanced processing methods are presented, which increase the penetration depth compared to the original processed data and enable a reliable extraction and detection of bottom points in deeper water bodies. The methodology is based on the analysis of correlated neighborhood information assuming a steady water bottom. The results confirm a significantly higher penetration depth with a high reliability of the additionally extracted water bottom points along with a larger coverage of the water bottom topography.
\end{abstract}

\section{INTRODUCTION}

Changes in water bottom topography can result from both, natural causes as well as human-induced activities. For instance, riverbeds underlie permanent natural change processes due to water currents and sedimentation. In addition, waterways are an important mode of transport and in Germany, for instance, the German Waterway and Shipping Administration (WSV) is responsible for their maintenance and an active waterway management (e.g. lowering of groin, clearing out of groin fields, widening of streaklines). For this purpose and for scientific applications, such as investigations of the German Federal Institute of Hydrology (BfG), reliable information about changes are required. Similarly, coastal areas undergo constant modifications affected by natural erosion processes. The monitoring of these morphologic changes are important for coastal protection purposes (Christiansen, 2016). For water bottom acquisition several measurement methods are available, such as echo sounders, airborne LiDAR bathymetry (ALB) and multimedia photogrammetry. Echo sounder sensors use the propagation of acoustic waves under water to determine the topography of water bottoms (similar to the laser scanning time of flight principle). It is an established method for a reliable acquisition of shallow water (e.g. riverbeds) and deep water (e.g. seabeds) bottom topographies in compliance with the minimum standards for hydrographic surveys (International Hydrographic Burea, 2011). The measurements are carried out from a vessel equipped with high-precision and high performance GNSS and IMU sensors for an accurate estimation of the position and orientation. With this measurement system a comprehensive data acquisition is time-consuming and expensive, but for deep water bodies without alternative. Airborne LiDAR bathymetry provides an area-wide measurement of the water bottom topography in

\footnotetext{
${ }^{*}$ Corresponding author
}

shallow waters, enabling an efficient monitoring of change processes. For that purpose, the water body is scanned by ALB systems with green laser wavelength, whereby a near-infrared laser can be optionally used to support water surface detection. A green laser pulse emitted at a known position and orientation interacts with all objects along the pathway. The backscattered

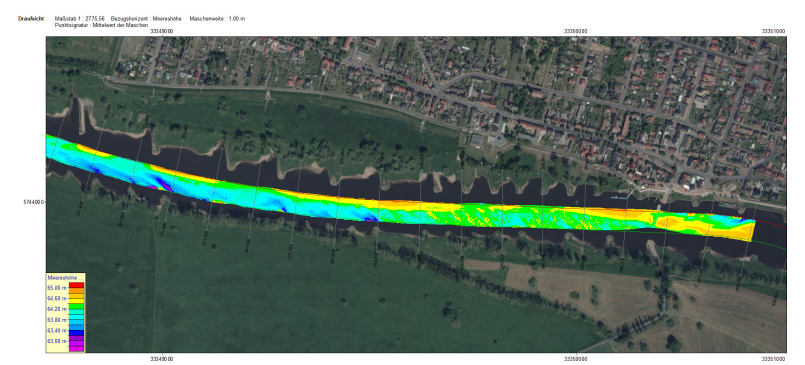

(a)

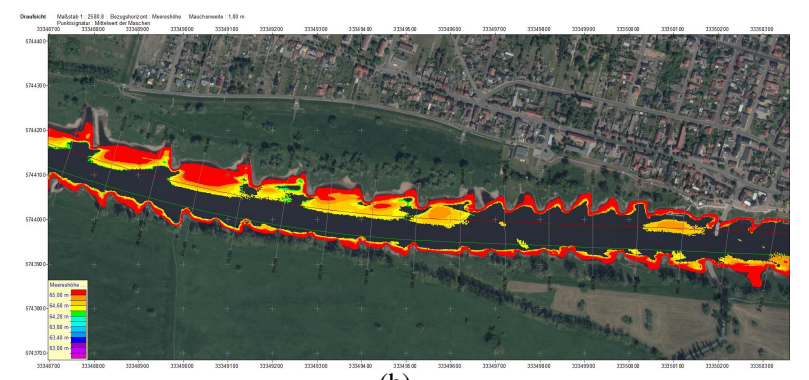

(b)

Figure 1. Water bottom points displayed color-coded by their height coordinate of a part of the river Elbe based on

(a) echo sounders and (b) ALB measurements. Source: German Federal Institute of Hydrology (BfG) and Waterways and Shipping Office Dresden (WSA) 


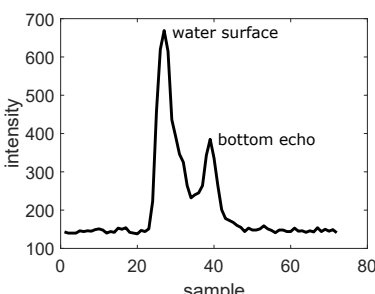

(a)

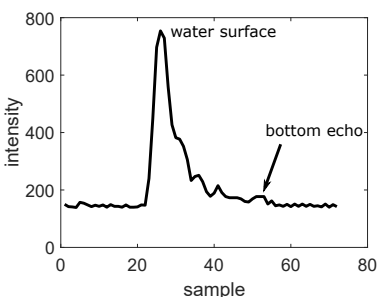

(b)
Figure 2. Full-waveforms with (a) two clearly visible signals for the water surface echo and the bottom echo as well as (b) water surface echo and bottom echo with less intensity.

signal is recorded by the sensor's detector using full-waveform digitization. Optionally, discrete 3D point coordinates can be derived by online data processing. Figure 1 shows echo sounders and airborne LiDAR bathymetry data of a part of the river Elbe. Due to the measurement principle the echo sounders data is limited to the waterway but has no data gaps. The result of the standard processed ALB measurements covers partially shallow waters areas, however, the water bottom is not captured completely. Obviously, the technology is mainly limited by water turbidity (Guenther, Goodman, 1978), preventing a reliable extraction of bottom points in turbid and deep waters, as the signal strength of the bottom echo is attenuated by the water column. In an ideal case, the full-waveform would show two clearly detectable signals, the water surface echo and the water bottom echo (fig. 2a). In turbid and deeper water bodies, many full-waveforms contain more than one potential weak bottom peak, so that the correctness of the detected bottom point cannot be guaranteed (fig. 2b). The goal of this contribution is the development of an extended processing approach considering correlated neighborhood information to increase (1) the penetration depth of the water body, (2) the coverage of captured water bottom topography and (3) the density of bottom points with a high reliability.

\section{STUDY AREA AND DATA ACQUISITION}

For the development and evaluation of the extended approach, measurement data of a part of the river Elbe between the German towns Klöden and Elster was used. Several subregions were selected, which are shown in Figure 3. The aerial survey of the region took place in spring 2015 with the bathymetry laser scanner RIEGL VQ-880-G. Additionally to the provided $3 \mathrm{D}$ point cloud, the backscattered signal at the receiver was digitized and recorded as a full-waveform (RIEGL, 2017). The recorded full-waveforms consist of 60 to 200 samples (intensity values) with a constant sampling interval of $0.575 \mathrm{~ns}$, corresponding to $6.45 \mathrm{~cm}$ sampling distance under water. A data density for the water body of up to 35 points $/ \mathrm{m}^{2}$ was achieved. The processing areas include the water body and the close shore area. For the development and execution of the extended methodology, the full-waveform data (fig. 4(a)), the (classified) point cloud resulting from standard processing (fig. 4(b)) and the trajectory (fig. 4(c)) was used. The evaluation of the results was carried out on the basis of the water bottom points obtained from the provider (fig. 4(d)) as well as the echo sounder data of the waterway (fig. 4(e)), acquired by the vessel ROSSLAU of the German Federal Waterways and Shipping Office Dresden (WSA) in spring 2015.

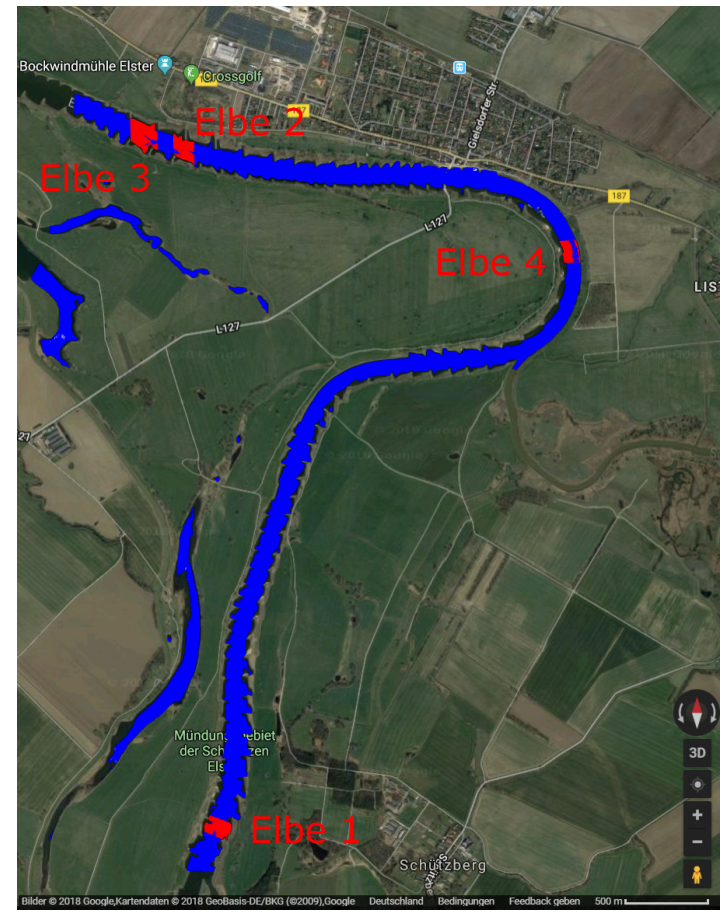

Figure 3. The study area (part of the river Elbe) including subregions for development and evaluation of the extended approach. (Map data (c)2018 Google)

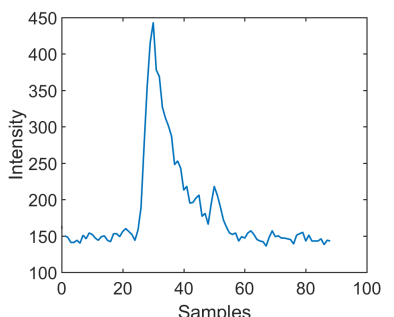

(a)

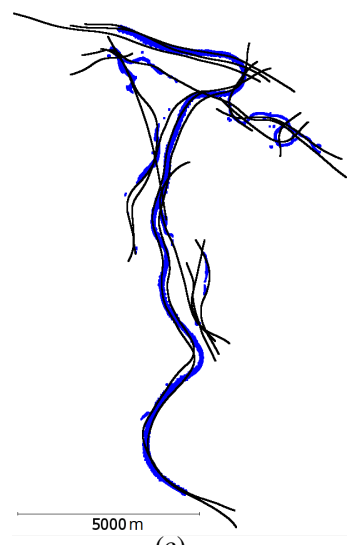

(c)

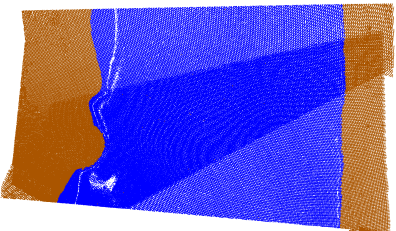

(b)

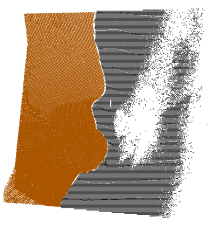

(d)

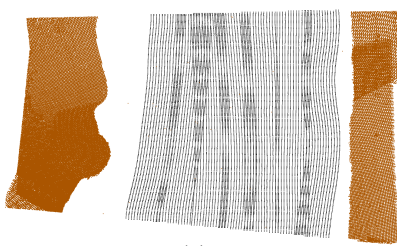

(e)
Figure 4. Data used for the analysis: (a) full-waveform data, (b) the points of water surface from the standard processing and (c) the trajectory. For the evaluation of the results of the extended approach (d) ALB bottom points from standard processing and (e) echo sounder data are used. 


\section{METHODS}

The basic idea of our approach is the limitation of the search range for the bottom echo in the full-waveforms based on an analysis of correlated neighborhood information for a reliable water bottom point extraction by using the data shown in section 2. The main data processing chain can be divided into three work steps (fig. 5). Firstly, the acquired data is mapped into a regular grid, whose size is adapted to the data density and the expected bottom topography (sec. 3.1). In the next step, an analysis of neighbor information related to the water depth is executed. In detail, all measured full-waveforms within a grid cell are processed by full-waveform-stacking techniques with the goal of defining a small search corridor, which is applied for water bottom echo detection in each full-waveform (sec. 3.2). Thereby averaging effect should be avoided. This is supported by information on the water depth of surrounding grid cells (sec. 3.3) to detect gross errors. Finally, the results from the neighborhood analysis are used for a reliable detection of the water bottom echo in every single full-waveform followed by the extraction of the water bottom point (sec. 3.4).

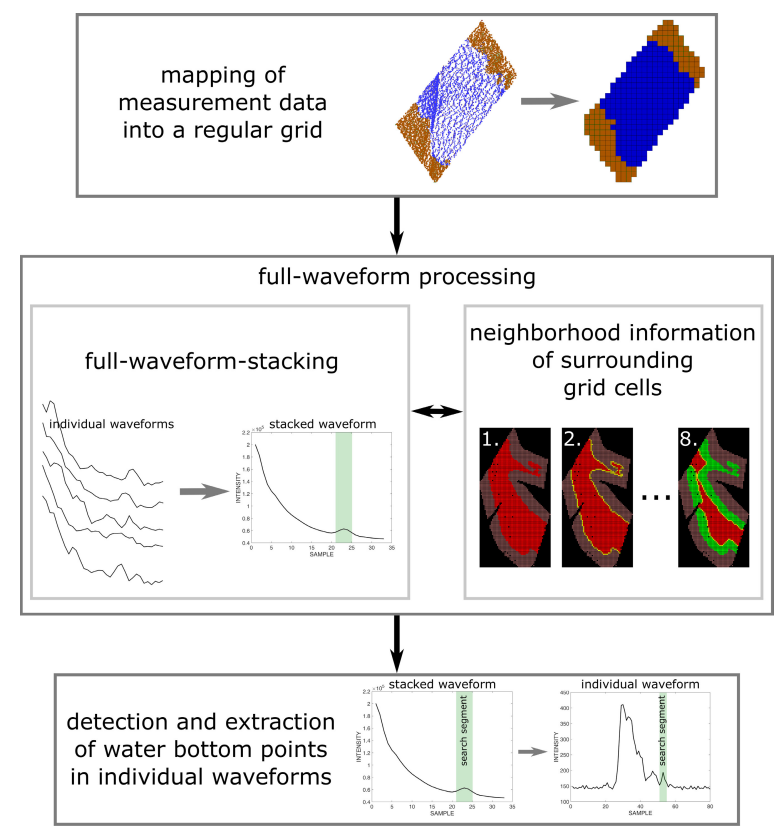

Figure 5. Workflow of extended processing of full-waveform data.

\subsection{Mapping of survey data}

The combined analysis and usage of neighborhood information is founded on the assumption that adjacent full-waveforms have similar or correlated properties (e.g. water bottom spots). A joint evaluation leads to a more reliable determination of water depths and a better identification of errors in the waveform. For this purpose, it is necessary to specify a suitable definition of neighborhood with regard to the number, spatial distance, and distribution of potentially adjacent measurement data. The present measurement campaign generally has a rather uniform point distribution and a high point density of up to 35 points $/ \mathrm{m}^{2}$. For the first development and evaluations, a simple mapping of the measurement values into a regular grid related to the water surface points was used (fig. 6). The point density and water bottom topography are important factors for the determination of the grid cell size. By expanding this, the

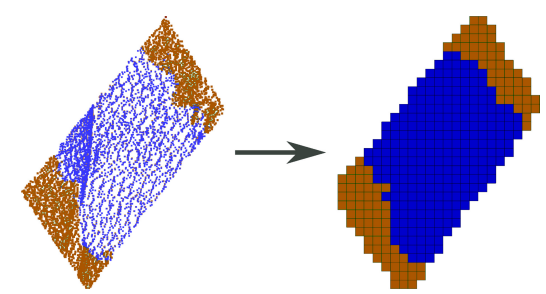

Figure 6. Mapping of measurement data to grid cells and their classification in land grid cells (brown) and water grid cells (blue).

number of full-waveforms and thus the reliability of the results increases. On the other hand, the correlation between the measured data decreases with increasing distance to each other. This can lead to a kind of smoothing effect, similar to a low-pass filtering. As a consequence, water bottom echoes might be identified potentially either incorrect or not at all. For the case presented here, empirical investigations revealed a suitable grid cell size between $2.5 \mathrm{~m} \times 2.5 \mathrm{~m}$ to $3 \mathrm{~m} \times 3 \mathrm{~m}$ resulting in 218 to 315 measurement values per grid cell. After creating a regular grid, the measured data is assigned to the grid cells depending on their spatial position. Therefore, the raster cells contain:

- the measured full-waveforms (fig. 4(a))

- original processed data obtained from the provider (fig. 4(b))

- origin of the pulse emission (fig. 4(c))

- classification (land/water) on basis of surface points (first echoes)

In the course of the processing, a rough approximation of the water depth based on the information of the surrounding grid cells (section 3.3) as well as a stacked full-waveform (section 3.2) are determined. The mapping method worked properly for the measurement campaign presented here. Nevertheless, the empirical determination of the grid cell size is not optimal and for the processing of water bottom topographies with different characteristics also not practicable. An automatic definition of the grid cell size based on the properties of the measured data is not trivial due to the great influence of the unknown water bottom topography. Further investigation on the spatial distribution of the data is required. The following variants can be considered:

- a flexible adaptation of the grid cell size during processing, depending on the depth of the water body and, if applicable, the degree of turbidity; low turbidity and low water depths lead to smaller grid cells and vice versa

- the determination of a predetermined number of the spatially closest measured data for each full-waveform to be processed

- the inclusion of all measurement data that has a maximum position distance in lateral direction at the water surface

\subsection{Full-waveform-stacking}

The determination of the water depth of a single grid cell is based on using correlated information of all full-waveform data, included in such a grid cell. A suitable and efficient method of 

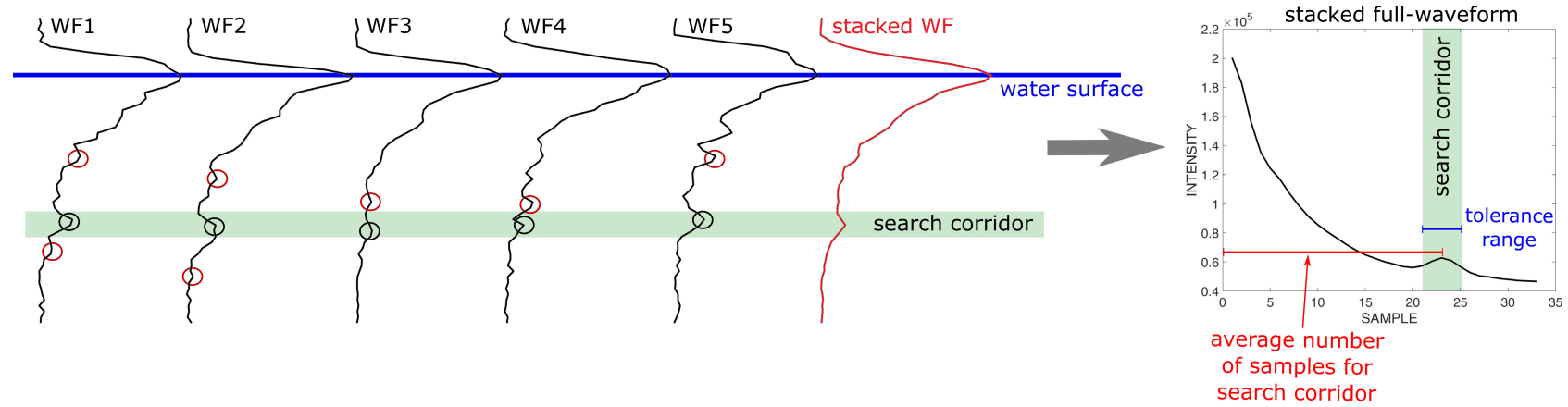

Figure 7. Basic principle of full-waveform-stacking applied to ALB pulse echo waveforms. The green marked area corresponds to the reduced search corridor for water bottom echoes in the original full-waveforms (black), based on the analysis of the stacked full-waveform (red).

combining the measured full-waveforms is a stacking approach, similar to the approaches presented by (Stilla et al., 2007), (Roncat, Mandlburger, 2016) and (Plenkers et al., 2013). For this purpose, all full-waveforms of the respective grid cell are firstly aligned to each other (for example, at the water surface peak) and secondly summed up (Figure 7). The analysis of such averaged full-waveforms reduces the random noise component of the returning signal and erratic backscatter effects within the water column. Recurring features (including water bottom points) with weak signal intensity are maintained and are in consequence better and more reliably detectable. As a result, a search corridor is defined by an average number of samples between the water surface and the assumed bottom plus a tolerance range, whereby the detection of the actual bottom echo in the individual full-waveform in section 3.4 is supported.

\subsection{Neighborhood relation between grid cells}

A reliable grid cell processing (sec. 3.2) requires approximate values for the water depth to avoid ambiguities in water bottom point extraction. For this purpose, we use water depth information of surrounding grid cells to derive control values for the single grid processing. In principle, the processing of the measuring area is executed iteratively, where in every iteration step solely grid cells with surrounding water depth information are processed. After every iteration there are more grid cells with depth information available, which are used for the next iteration step. As there are no water grid cells with water depth information in the initial iteration step, the processing starts at the grid cells on the shore. The water depth of the land grid cells is set to $0.0 \mathrm{~m}$ for the calculations. Based on this, the remaining grid cells are processed iteratively towards the center of the water body (fig. 8) until all grid cells have been processed.
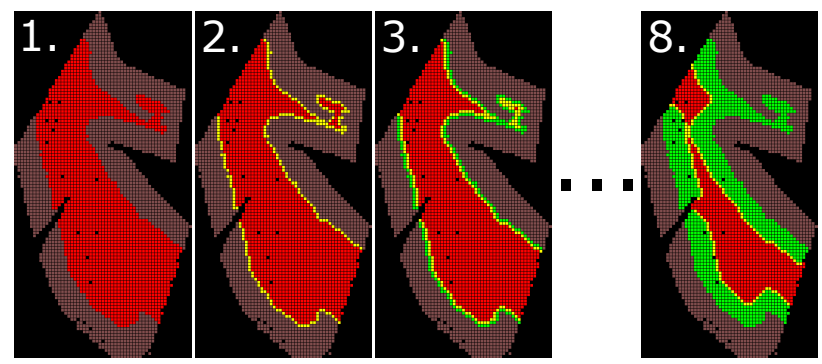

Figure 8. Iterative processing of the measuring area from shore to water center. Brown $=$ land grid cells. Yellow $=$ currently processed grid cell. Red $=$ non processed grid cells. Green $=$ processed grid cells .

\subsection{Detection of bottom echo and extraction of bottom point}

Based on the results of the full-waveform-stacking, the most likely position of the water bottom echo can be detected in each measured full-waveform. For this purpose, each full-waveform is analyzed in the determined search corridor and, if present, a local maximum is detected. The isolation of a small search corridor in the original full-waveform based on the full-waveform-stacking is advantageous here, as especially on rough water bottom - that smoothing effects by the full-waveform-stacking method are minimized. Finally, the bottom point coordinates can be calculated by using the known beam geometry and the number of samples or running time of the laser beam between the water surface and the water bottom.

$$
\mathbf{P}_{w b}=\mathbf{P}_{w s}+t_{2} \cdot c_{w} \cdot \mathbf{X}_{2}
$$

Figure 9 and equation 1 illustrate the principal beam geometry from the origin of pulse emission $\mathbf{P}_{l s}$ via the water surface $\mathbf{P}_{w s}$ to the water bottom point $\mathbf{P}_{w b}$. The direction vector $\mathbf{X}_{1}$ results from the origin of the pulse emission $\mathbf{P}_{l s}$ and the water surface point $\mathbf{P}_{w s}$. The coordinates of the water surface point are taken from the original processed data obtained from the provider. On the basis of the direction vector $\mathbf{X}_{1}$ as well as the local water surface normal, calculated from neighboring water surface points (Richter et al., 2018), the refraction correction according to the Snell's Law is applied. Consequently, the new propagation direction $\mathbf{X}_{2}$ of the laser beam can be determined. With the transit time $t_{2}$, adjusted in accordance with the propagation velocity from light in water $c_{w}$, the water bottom point is calculated.

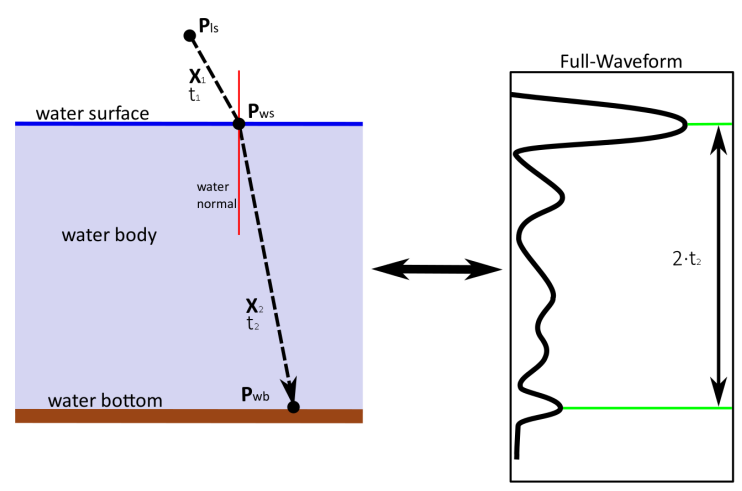

Figure 9. Schematic illustration of water bottom point calculation. 


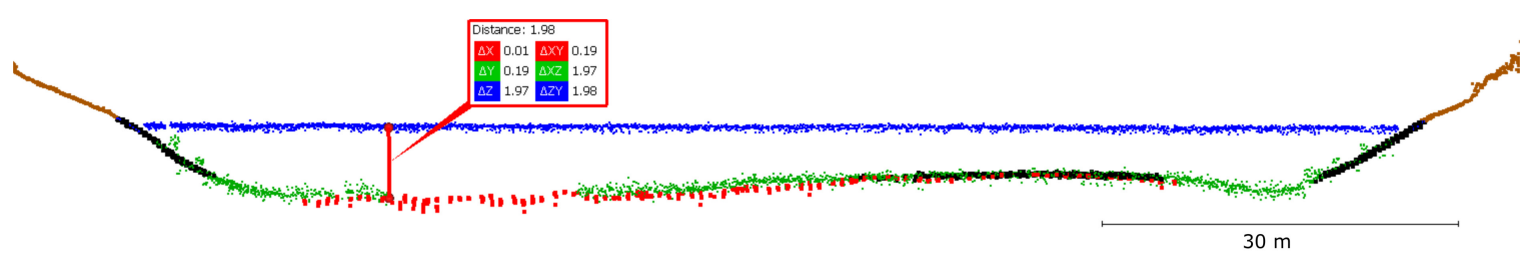

Figure 10. Profile of the riverbed of the river Elbe. Black = Points from online-waveform-processing. Blue = water surface points. Red = points from echo sounder reference. Green = points from full-waveform-stacking method.

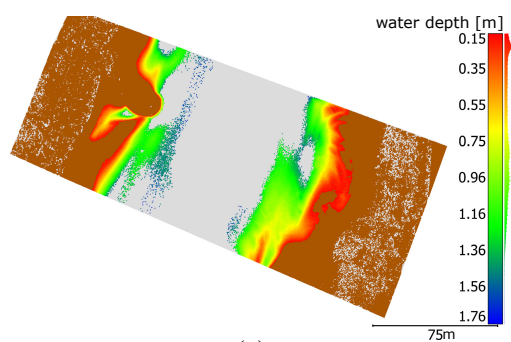

(a)

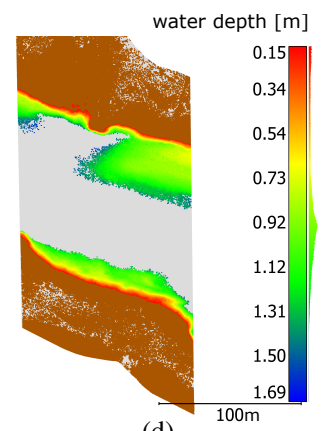

(d)

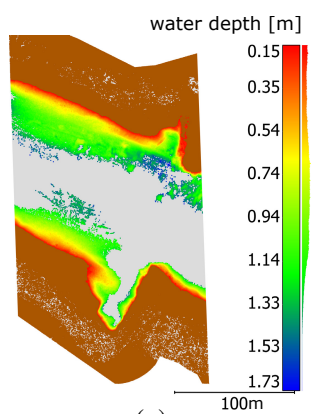

(g)

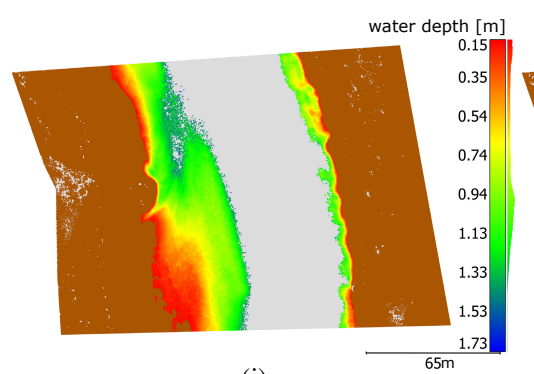

(j)

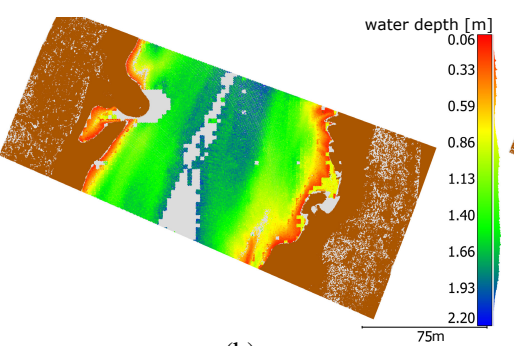

(b)

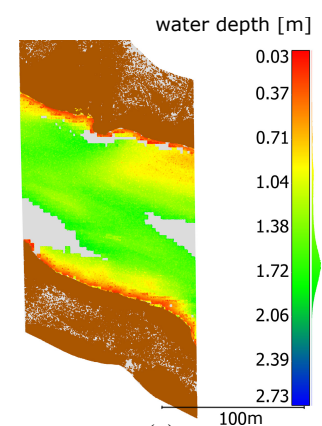

(e)

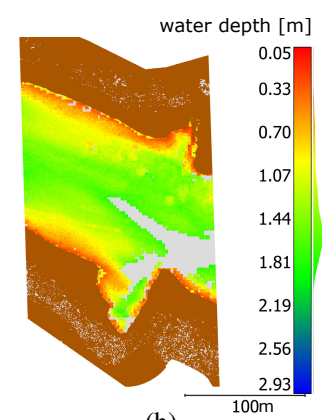

(h)

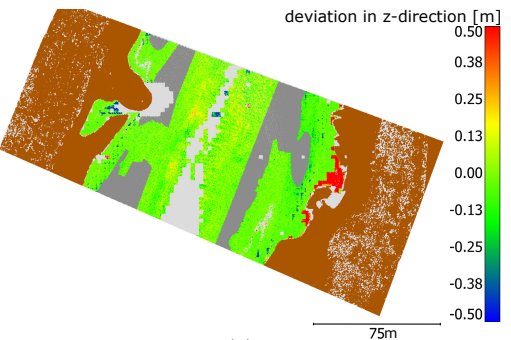

(c)

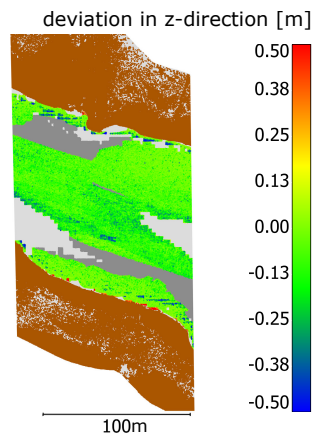

(f)

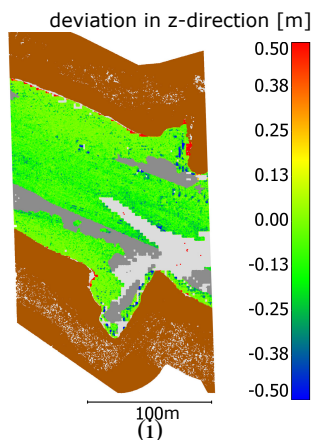

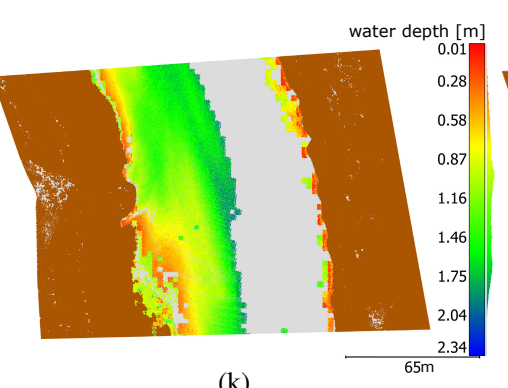

$(\mathrm{k})$

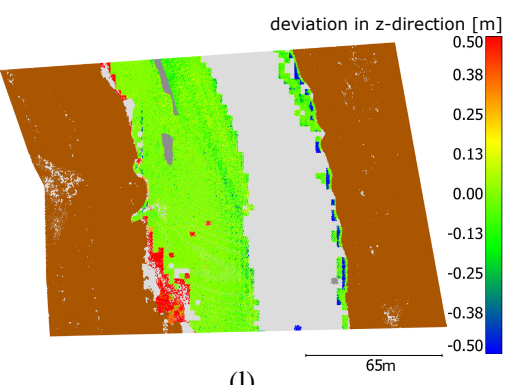

(1)

Figure 11. Evaluation of the results of Elbe 1 (a)-(c), Elbe 2 (d)-(f), Elbe 3 (g)-(i) and Elbe 4 (j)-(l). The left column shows the standard processed points with their water depth (color-coded), the middle shows the new processed bottom points with their water depth (color-coded) and the right column shows the results of the comparison of both data related to the $\mathrm{z}$-direction, where the dark-grey areas are incomparable new processed points (no comparative data available).

Brown points are land points. 
Table 1. Line 1-2 show the mean deviation value and the $R M S$-deviation of the height component between new recognized points and the comparative data. Line 3-4 present the number of points with a max. height-deviation of

$0.25 \mathrm{~m}$ related to the number of all recognized points and the percentage.

\begin{tabular}{c|rrrr}
\hline & Elbe 1 & Elbe 2 & Elbe 3 & Elbe 4 \\
\hline mean deviation & $0.039 \mathrm{~m}$ & $0.046 \mathrm{~m}$ & $0.042 \mathrm{~m}$ & $0.058 \mathrm{~m}$ \\
RMS & $0.13 \mathrm{~m}$ & $0.10 \mathrm{~m}$ & $0.10 \mathrm{~m}$ & $0.18 \mathrm{~m}$ \\
\hline max. $0.25 \mathrm{~m}$ & $159374 / 169390$ & $514209 / 529970$ & $637071 / 659140$ & $124951 / 136289$ \\
& $94.09 \%$ & $97.03 \%$ & $96.65 \%$ & $91.68 \%$ \\
\hline
\end{tabular}

\section{RESULTS}

In this section, the results of the processed subregions are presented and evaluated in regard to accuracy and reliability. For this, reference data with a significantly higher accuracy would be necessary but are not available. The data from standard processing and the echo sounder measurements probably have a similar error budget as the results. Consequently, they are thus used as comparative data. Furthermore, accuracy requirements have to be defined to assess the results regarding the reached accuracy. Therefor, the minimum standard for hydrographic surveys of $0.25 \mathrm{~m}$ released by the International Hydrographic Organization (International Hydrographic Burea, 2011) is used for the evaluation of the results.

The measured data was processed with a grid cell size of $2.5 \mathrm{~m}$ $\times 2.5 \mathrm{~m}$ with up to 218 points per grid cell. An initial visual analysis of the results using a profile of the study area (fig. 10) illustrates that the new bottom points represent large parts of the water bottom well and reliably with penetration depths of up to approximately $1.97 \mathrm{~m}$, compared to $1.50 \mathrm{~m}$ for the standard processing. For the accuracy and reliability analysis of the results, the mean deviation value and the root mean square deviation $(R M S)$ of the height components between results and comparative data is used (tab. 1, line 1-2). In addition, the reliability of the newly detected water bottom points is evaluated by determining the percentage of points, which deviation's is equal or less than $0.25 \mathrm{~m}$ in the height component (tab. 1, line 3-4). The analysis of the results of the study areas confirms a good representation of the water bottom topography by the newly detected ground points. The mean deviations in $\mathrm{z}$-direction are all positive in a range of $3.9 \mathrm{~cm}$ and $5.8 \mathrm{~cm}$, which means the new bottom points are by trend above the comparative points (considering the evaluation proceeded in a north-east-down coordinate system). The RMS deviations with respect to the height component to the comparative data are between $0.10 \mathrm{~m}$ and $0.18 \mathrm{~m}$ (fig. 11 and tab. 1). The percentage of points which do not exceed a height deviation of $0.25 \mathrm{~m}$ is $91.68 \%$ to $97.03 \%$. The good height accuracy and the high reliability as well as an extended penetration depth of up to $1.95 \mathrm{~m}$ lead to a more extensive coverage of the water bottom topography (fig. 11).

\section{FUTURE EXTENSION OF THE NEW APPROACH - CORRECTION OF SIGNAL ATTENUATION}

The attenuation of the green laser pulse is mainly induced by absorption and scattering effects at water molecules in the water column. Depending on the degree of turbidity and the water depth, the water bottom echoes can become too weak for the reliable detection in a single waveform processing. To compensate the attenuation effects, Richter et al. (2017) presents an individual pulse history based attenuation correction for each laser pulse using the differential backscatter cross section. The basic idea of the correction method is the step-wise amplification of the signal intensities with an appropriate correction factor. The correction factor depends on the pulse history and is derived from the data set itself (fig. 12). However, the noise is amplified too, which is a disadvantageous factor for the detection of bottom points. In further work the method for correction of signal attenuation will be integrated and tested in our neighborhood-based approach.
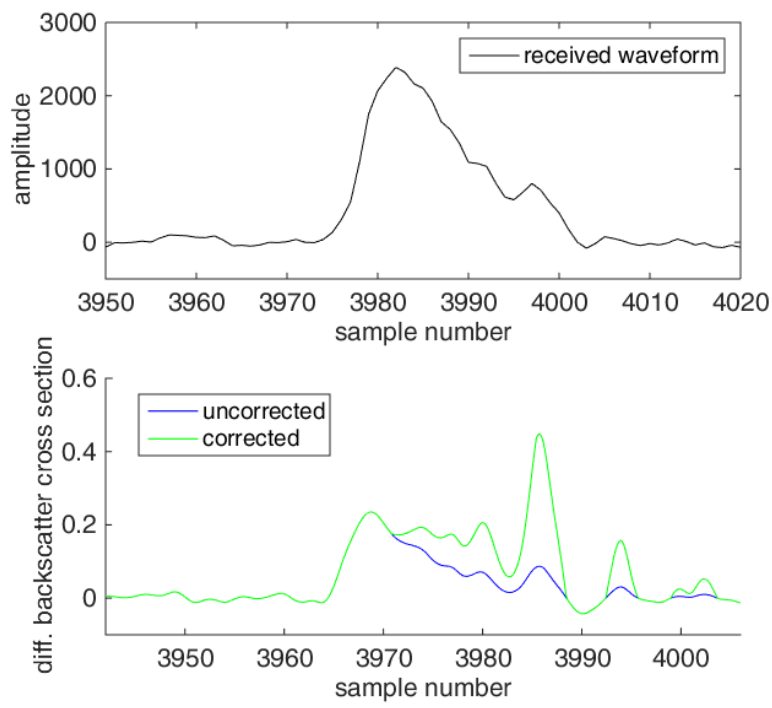

Figure 12. Recorded waveform (top), differential backscatter cross section (bottom blue) and attenuation-corrected differential backscatter cross section (bottom green) (Richter et al., 2017).

\section{CONCLUSSION AND FUTURE WORK}

The paper presents a novel approach for full-waveform processing of airborne LiDAR bathymetry data for detection and extraction of water bottom points. The basic idea is the inclusion of neighborhood information of each full-waveform by using full-waveform-stacking techniques. As a result of the full-waveform-stacking, there is a small search corridor applied for the water bottom detection. As a consequence of this procedure, averaging or smoothing effects during the extraction of the water bottom points can be minimized. The objective of an increased penetration depth and a higher data density, and thus a complete detection of the riverbed with a high reliability has been achieved to a high degree. The results demonstrate the good applicability of the developed method to the given data. The increase of the penetration depth from approx. $1.50 \mathrm{~m}$ up to $1.95 \mathrm{~m}$ corresponds to an improvement of $30 \%$. This directly leads to a larger coverage of the water body topography by the newly recognized points. The evaluation with the comparative data of the riverbed proves 
the reliability by using full-waveforms as data basis. About $96.04 \%$ of the detected points did not exceed a height deviation of $25 \mathrm{~cm}$ from the comparative data. The RMS deviations in the height component for all newly detected points was $0.11 \mathrm{~m}$ and shows a good height accuracy. However, the gap to the echo sounder measurements could not always be closed, since penetration depths greater than $1.95 \mathrm{~m}$ would have been necessary. Nevertheless, the processed data represent an improvement or extension of the data set resulting from standard processing. The methods developed offer potential for further adjustments and improvements so that the following goals are pursued in future work:

- adaptive neighborhood definition when selecting full-waveforms for full-waveform-stacking

- investigations of the potential of an alternative spatial division of the processing areas in conjunction with the full-waveform-stacking (see section 3.1)

- improving of the point detection and extraction of more dynamic water body topographies (e.g shore areas)

- developing additional strategies or statistical tests to increase the reliability for extracting water bottom points

- further investigations regarding the use of attenuation corrected data for an additional increase of the penetration depth (see section 5)

- extension of the approach to consider wave pattern induced systematic coordinate errors (Westfeld et al., 2017)

Furthermore, the potential of the advanced processing methods presented here for application to maritime waters will be investigated. In maritime waters, a lower turbidity and thus a higher penetration depth can often be assumed. In addition, other conditions such as the influence of maritime waves will represent new challenges. It is to be expected that the transfer of the presented processing methods to maritime ALB data leads to an improvement of the recording of seabed topographies.

\section{ACKNOWLEDGEMENTS}

The research project on airborne LiDAR bathymetry is funded by the German Research Foundation (DFG). We also thank the German Federal Institute of Hydrology for the research assignment of a pilot study, which allowed intensive practical applications of the developed methods. Additionally, we thank the Waterways and Shipping Office Dresden (WSA) for the provision of the survey data, and the company Airborne Hydro Mapping GmbH (AHM) for the good cooperation in the preparation of the survey data.

\section{REFERENCES}

Christiansen, L., 2016. New techniques in capturing and modelling of morphological data. Hydrographische Nachrichten, HN 105, 20-23.

Guenther, G. C., Goodman, L. R., 1978. Laser applications for near-shore nautical charting. Ocean Optics $V, 160$, International Society for Optics and Photonics, 174-184.
International Hydrographic Burea, 2011. Manual on Hydrography, Publication C-13. International Hydrographic Organization.

Plenkers, K., Ritter, J. R., Schindler, M., 2013. Low signal-to-noise event detection based on waveform stacking and cross-correlation: application to a stimulation experiment. Journal of seismology, 17, 27-49.

Richter, K., Mader, D., Westfeld, P., Maas, H.-G., 2018. Numerical simulation and experimental validation of wave pattern induced coordinate errors in airborne LiDAR bathymetry. International Archives of the Photogrammetry, Remote Sensing \& Spatial Information Sciences, 42.

Richter, Katja, Maas, Hans-Gerd, Westfeld, Patrick, Weiß, Robert, 2017. An approach to determining turbidity and correcting for signal attenuation in airborne lidar bathymetry. PFG-Journal of Photogrammetry, Remote Sensing and Geoinformation Science, 85, 31-40.

RIEGL, 2017. Waveform Extraction Library. Technical report, RIEGL Laser Measurement Systems GmbH.

Roncat, Andreas, Mandlburger, Gottfried, 2016. Enhanced detection of water and ground surface in airborne laser bathymetry data using waveform stacking. EGU General Assembly Conference Abstracts, 18, 17016.

Stilla, U., Yao, W., Jutzi, B., 2007. Detection of weak laser pulses by full waveform stacking. International Archives of Photogrammetry, Remote Sensing and Spatial Information Sciences, 36, W49A.

Westfeld, Patrick, Maas, Hans-Gerd, Richter, Katja, Weiß, Robert, 2017. Analysis and correction of ocean wave pattern induced systematic coordinate errors in airborne LiDAR bathymetry. ISPRS Journal of Photogrammetry and Remote Sensing, 128, 314-325. 